\title{
Experimental investigation of flow through a bileaflet mechanical heart valve
}

\author{
J. Mejia \& P. Oshkai \\ Department of Mechanical Engineering, University of Victoria, Victoria, \\ $B C$, Canada
}

\begin{abstract}
Turbulent flow downstream of a bileaflet mechanical heart valve is investigated using digital particle image velocimetry. Evolution of flow structures during the systole and diastole phases of a typical cardiac cycle is characterized by obtaining global flow velocity measurements in multiple cross-sections of the flow field. Instantaneous and time-averaged patterns of flow velocity, vorticity, and streamline topology are used to illustrate the interaction between the unsteady vortices that results in elevation of shear stress levels. This imagebased approach can potentially lead to the development of methods for the control of platelet activation and provides insight into the underlying flow physics.
\end{abstract}

Keywords: bileaflet mechanical heart valves, particle image velocimetry, in-vitro.

\section{Introduction}

Currently, about 180,000 prosthetic heart valves are implanted each year worldwide, Yoganathan et al. [1]. Mechanical Heart Valves (MHVs) are relatively durable but strongly associated with thromboembolisms, which often result in ischemic attacks and stroke, Yin et al. [2]. It is believed that thrombi are caused by flow phenomena not characteristic of physiological conditions, Bluestien et al. [3]. The flow phenomena that receive the most attention in this respect include: jet-like flow regions, regions of elevated shear stress, flow separation regions, shed vortices, and turbulence characteristics, Yin et al. [2] Despite the recent advances in the field, thromboembolisms occur in 
approximately $3 \%$ of all replacement operations involving MHVs, Edmunds et al. [4].

On the other hand, biological valves exhibit calcification, and durability problem $[1,5]$. However, biological prosthetic heart valves do not require the use of blood thinners to be administered to the patient, making them an attractive alternative to MHVs. A number of experimental and numerical studies have been undertaken in order to address problems with both biological and mechanical heart valves.

\subsection{Experimental studies}

Significant effort has been devoted to developing realistic laboratory models capable of simulating the fluid mechanic characteristics of the human heart while allowing optical access for the purpose of flow visualization. The complexity of the biological system calls for a compliant setup that not only preserves geometric similarity but also models the pumping cycle. Anatomically correct mock-ups of the aorta have been successfully manufactured and investigated. Scotten and Walker [6] developed a compliant model of the left heart, which allowed the simulation of an arbitrary cardiac cycle and provided optical access to valves in both the aortic and the mitral positions. Several studies have been conducted using this setup, including the development of a new technique to measure the projected dynamic area of prosthetic heart valves, Scotten and Walker [6] Marassi et al. [7] developed an artificial heart valve test bench specifically designed to employ particle image velocimetry (PIV). Test benches for digital PIV and stereo PIV were developed. Although slightly different both designs consisted of a valve mounted in a Plexiglas channel. In both cases the channel was surrounded with a Plexiglas chamber in order to minimize optical distortions. His design delivered high versatility in changing and controlling the flow parameters and good optical access for visualization experiments.

It has been suggested that among the many possible causes of thromboembolisms, vortex shedding from the leaflets of MHVs is among the most critical, Yoganathan et al. [1]. The shed vortices are associated with regions of high shear stresses in the flow. Blood components (e.g. platelets) can become entrapped in these vortices for relatively long periods of time, increasing their chance of becoming activated.

Several studies have focused on the fundamental fluid dynamics of MHVs in order to provide insight into the flow phenomena. Castellini et al. [8] employed PIV techniques to obtain flow velocity measurements upstream and downstream of a bileaflet valve. A symmetric flow pattern including a large separation region behind each leaflet was observed. Furthermore, the authors observed regions of high velocity between the leaflets as well as between each leaflet and the channel walls, which represented the walls of the aorta. Vortex shedding from the trailing edge of each leaflet was also documented.

Bluestien et al. [9] performed a study of pulsatile flow through a bileaflet MHV mounted in a non-compliant aorta model. A technique of digital particle image velocimetry (DPIV) was used to characterize the flow. The authors assumed (based on the results of Lamson et al. [10]) that no single phase of the 
heart cycle (i.e. opening, fully open, closing, or fully closed) contributed significantly more to the formation of thrombi than any other phase. However, a number of other investigations have focused on particular phases of the cardiac cycle. In fact, during the 1970 s and 1980s, most of the studies implicitly assumed that blood damage occurs predominantly during forward peak flow through the valve. This assumption is intuitive, as the flow rate is at its maximum when the valve is fully open, but later studies have challenged the validity of this assumption. Manning et al. [11] investigated the regurgitant flow field of a bileaflet MHV using PIV under physiological pulsatile flow conditions. The authors found strong jets emerging from the two hinges, and two weaker jets originating from the regions of transition between the tightly sealed central plane and the hinge region. Maximum viscous shear stresses were found to be of the order of $20 \mathrm{~N} / \mathrm{m}^{2}$, which is below the shear stress traditionally associated with the onset of hemolysis. However, other studies have found Reynolds's stresses above the threshold for platelet activation, Meyer [12].

\subsection{Numerical studies}

Numerical methods play a fundamental role in the research of fluid dynamics. For example, numerical simulations can provide detailed three-dimensional, time-resolved information about a flow. Hence, an integrated approach involving both experimental and numerical techniques is often required to fully characterize a fluid flow problem.

Since platelet activation is closely linked to the occurrence of thromboembolisms, a number of studies have focused on the effect of prosthetic heart valves on platelet activation. If a platelet reaches a certain threshold of cumulative exposure to high shear stress, it becomes activated. The platelet activation state (PAS) was studied for monoleaflet and bileaftlet valves by Yin et al. [2]. The authors considered both shear stress magnitude and time of exposure of a given platelet to the elevated shear stress to determine the PAS. In order to determine the amount of activated platelets numerically, tracers were introduced to the calculated flow. The exposure of a tracer to shear stresses over a period of time was then evaluated. Monoleaflet valves were found to cause less PAS compared to bileaflet MHVs. Furthermore, the results showed that virtually no PAS existed in a control case when the valve was removed from the flow field.

Bluestein et al. [9] identified vortex shedding as a possible mechanism for the activation of platelets. "The shed vortices also provide the flow conditions that promote the formation of larger platelet aggregates... Following activation, platelets will release their granule constituents and provide positive feedback reactions of coagulation. Platelet aggregates will increase the efficiency of the reaction, eventually resulting in the formation of free emboli."

\section{Experimental system and techniques}

The experiments for the current study were conducted using a Carbomedics bileaflet mechanical heart valve. The complete flow loop, illustrated in Figure 1, 
consisted of a Plexiglas duct, the test section, flexible tubing, a water reservoir, and a pump. A Plexiglas test section was designed to provide optical access to the region downstream of the heart valve. The valve $(20.39 \mathrm{~mm}$ in diameter) was mounted in the Plexiglas duct. A constant pressure head corresponding to a Reynolds number of 9500 was maintained by a 1/6 hp pump. This Reynolds number corresponds to a net positive average flow speed of approximately $1.35 \mathrm{~m} / \mathrm{s}$, corresponding to the peak systolic flow rate of a normal heart with a cardiac output of $5 \mathrm{l} / \mathrm{min}$.

The test section was located $254 \mathrm{~mm}$ downstream of the main duct inlet. A honeycomb flow straightener was employed directly downstream of the inlet. To minimize optical distortions, a prismatic acrylic chamber filled with the working fluid surrounding the test section was employed during the experiments. A detailed schematic of the test section is provided in Figure 2.

The coordinate system shown in Figure 3 is used in the analysis presented in later sections. The valve's horizontal axis of symmetry is defined as $z=0$ and the valve's vertical axis of symmetry is defined as $y=0$. The $x=0$ coordinate corresponds to the downstream edge of the valve housing. Flow measurements are not performed within the first $10 \mathrm{~mm}$ downstream of the valve housing due to optical inaccessibility.

In order to characterize the three-dimensionality of the flow several parallel planes, positioned along the vertical axis, are used as the data acquisition planes (DAP). The set consisted of three parallel and horizontal planes positioned at 0 , 2.6, and $8 \mathrm{~mm}$ along the $\mathrm{z}$-axis (DAP A through $\mathrm{C}$ ), as shown in Figure 3.

Quantitative flow visualization is accomplished by employing DPIV. Titanium dioxide seeding (nominal diameter of $1 \mu \mathrm{m}$ ) serves as tracer particles for the forward flow phase experiments. Images of the particles, which are illuminated by a planar laser sheet, are captured by a high-resolution digital camera. These images are then processed on a computer to yield global instantaneous flow velocity measurements as well as maps of vorticity, streamline topology and time-averaged flow parameters. The displacements of the particles are recorded as a pair of images, each exposed once. The recorded particle displacement field is measured locally across the whole field of view of the images, scaled by the image magnification and then divided by the known laser time delay to obtain flow velocity at each point. The charge-coupled device (CCD) camera is positioned perpendicular to the plane of the light. Depending on the flow velocity and the magnification factor of the camera lens, the time delay between the two pulses is chosen such that adequate displacements of the particle images on the CCD were obtained. From the time delay between the two illuminations and the displacement of the tracers, velocity vectors are calculated.

For the present study, a lens with a focal length of $60 \mathrm{~mm}$ is used in conjunction with a 1376 × 1040 pixel CCD camera to provide a physical resolution of the velocity vector field of 4 vectors per square millimeter.

The PIV measurements are acquired at a time interval of $0.204 \mathrm{~s}$, which provided spacing in time appropriate for obtaining random samples for the calculation of averaged turbulence statistics. A total of 1000 images are acquired 
and used in the calculation of the following time-averaged parameters: flow velocity $\langle\underline{v}\rangle$, out-of-plane vorticity $\left\langle\omega_{\mathrm{Z}}\right\rangle$, root-mean-square values of velocity components $\left\langle\mathrm{u}_{\mathrm{rms}}\right\rangle$ and $\left\langle\mathrm{v}_{\mathrm{rms}}\right\rangle$, and Reynolds stress correlation $\left\langle\mathrm{u}^{\prime} \mathrm{v}^{\prime}\right\rangle$.

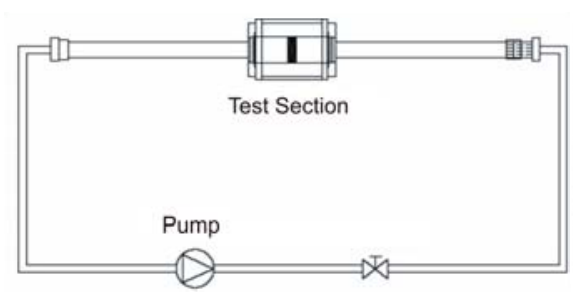

Figure 1: Complete flow loop.

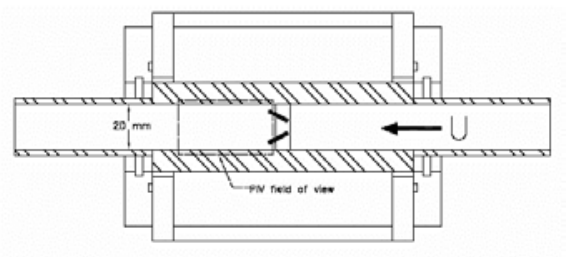

Figure 2: Close-up of the test section.

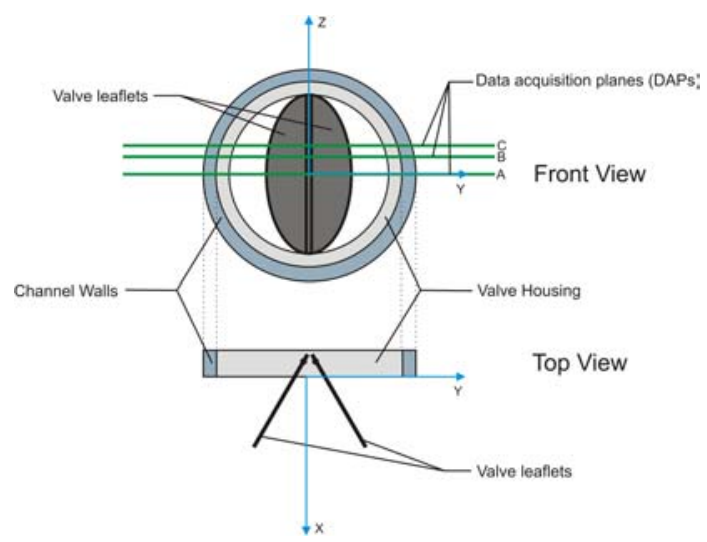

Figure 3: Definition of coordinate system.

\section{Results}

All results presented here correspond to a steady unidirectional turbulent inflow from right to left. The leaflets of the fully open valve are indicated by the red shaded areas outlined in white. The regions between the leaflets as well as those directly above the upper leaflet do not show velocity vectors due to optical inaccessibility.

\subsection{Instantaneous flow patterns}

Plots of instantaneous velocity, $\langle\underline{\mathrm{v}}>$, and the associated out-of-plane vorticity, $<\omega_{\mathrm{z}}>$, corresponding to DAP A are shown in Figure 4.

Generally, the following flow structure is observed downstream of the valve in each of the three horizontal data acquisition planes (DAPs A-C): Shear layers are formed at the tips of both leaflets. The outer shear layers, which form at the trailing edges of the leaflets, roll up into vortices that are shed. It is traditionally 
assumed that the inner shear layers, which form at the leading edges of the leaflets, roll into vortices that stay attached to the leaflet surface. Present observations indicate that for the case of unidirectional flow through a fully-open valve, vortex shedding occurs from both the inner and outer tips of the leaflets. The four shear layers define two wake regions, one behind each leaflet. A high velocity jet-like flow region exists between the two wakes. This high-velocity flow will be referred to here as the central jet. The central jet is unsteady in nature. It exhibits a large-scale, low frequency oscillation in the transverse direction. This phenomenon occurs simultaneously with the vortex shedding from the tips of the leaflets. Figures 4(a) through 4(c) illustrate three characteristic phases of this oscillation that correspond to DAP A. These phases are referred to as dominant lower wake regime Figure 4(a), symmetric wake regime Figure 4(b), and dominant upper wake regime Figure 4(c).

Figure 4(a) corresponds to the instant in time when the wake from the lower leaflet dominates the flow field. The velocity vector plot of Figure 4(a) shows that the low velocity region corresponding to the lower wake occupies a significantly larger area compared to the upper wake. In addition, the velocity plot shows a pronounced upward deflection of the central jet.

In comparison, the lower wake exhibits significantly higher levels of vorticity than the upper wake. The vorticity contour plot of Figure 4(a) shows that the outer shear layer of the bottom leaflet contains three well-defined negative vortices that were shed from the trailing edge of the leaflet. The distance between these negative vortices indicates that their shedding frequency is similar to that of the vortices in the inner shear layer. Moreover, the vortices in the outer and inner shear layers of the lower leaflet retain substantial levels of circulation up to $20 \mathrm{~mm}$ downstream of the valve.

In contrast to the lower leaflet, the outer and inner shear layers of the upper leaflet are located significantly closer to each other. In particular, the negative vortices of the inner layer develop close to the leaflet surface and interact with the positive vortices of the outer shear layer by forming counter-rotating vortex pairs. As the counter-rotating vortices move downstream the interaction between them results in a rapid decrease of their circulation levels. Rapid dissipation of vorticity due to shear layer interaction results in the early collapse of the upper wake, which extends only $10 \mathrm{~mm}$ downstream of the valve. This collapse is accompanied by the upward deflection of the inner shear layer of the lower leaflet.

The next characteristic phase of the flow oscillation cycle is referred to as the symmetric wake regime and is illustrated in Figure 4(b). During this phase, the four shear layers do not exhibit significant transverse deflections. Both the upper and lower wakes contain comparable vorticity levels up to $10 \mathrm{~mm}$ downstream of the valve. The symmetric wake regime corresponds to the transition between the dominant lower wake phase of Figure 4(a) and the dominant upper wake phase, which is illustrated in Figure 4(c).

Patterns of instantaneous flow velocity and out-of-plane vorticity shown in Figure 4(c) correspond to the flow oscillation phase that is opposite to the dominant lower wake regime of Figure 4(a). The velocity field of Figure 4(c) 
shows that the wake region, which forms behind the upper leaflet, dominates the flow field. Rapid dissipation of positive vorticity in the inner shear layer of the lower leaflet is accompanied by a large-scale downward deflection of the inner shear layer of the upper leaflet and the associated jet-like flow in the middle of the channel.

It is evident that the frequency of the large-scale flow oscillation that is represented by the sequence of images in Figures 4(a) through 4(c) is substantially lower than the frequency of the vortex shedding from the tips of the leaflets. In fact, at least four small-scale vortices are shed from the leaflet tips during a typical large-scale oscillation cycle described above.

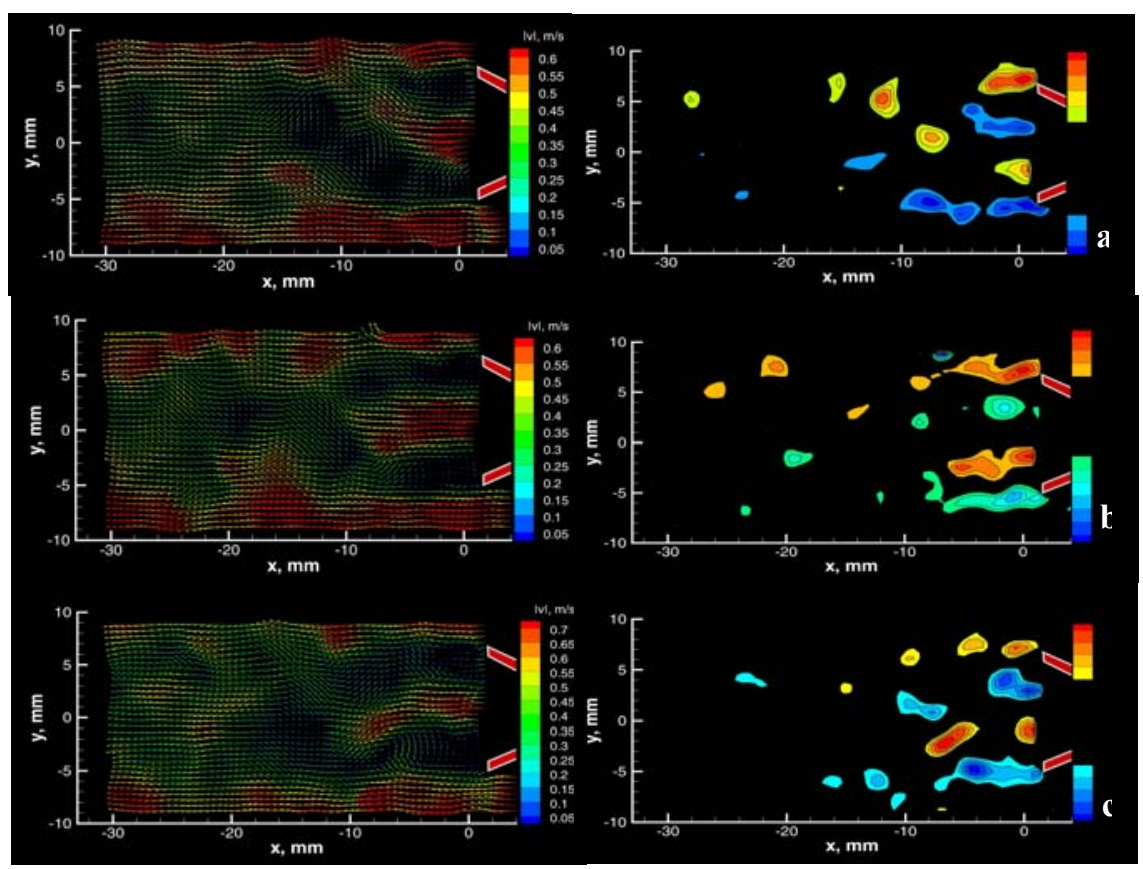

Figure 4: Instantaneous velocity field (left) and out-of-plane vorticity (right) for: (a) the dominant lower wake regime of DAP A (b) the symmetric wake regime of DAP A (c) the dominant upper wake regime of DAP A.

\subsection{Time-averaged flow patterns}

All images presented in this section are a result of ensemble-averaging of 1000 instantaneous images similar to those presented in the previous section.

The structure of the wake downstream of the bileaflet MHV, corresponding to DAP A, is clearly evident in the plot of time averaged vorticity $\left\langle\omega_{z}\right\rangle$, which is shown in Figure 5(a). The dominant feature of the flow field downstream of the valve is the presence of four separated shear layers that form at the tips of the 
leaflets. The outer shear layers form at the trailing tips of the upper and lower leaflet. They are indicated in the plot of out-of-plane vorticity of Figure 5(a) by the regions of high positive and negative time-averaged vorticity respectively. These layers extend approximately $14 \mathrm{~mm}$ downstream of the valve. The inner shear layers, which form at the leading tips of the leaflets, exhibit high levels of time-averaged vorticity of the opposite sign, relative to the outer shear layers. Due to the highly unsteady nature of the inner shear layers, the time-averaged vorticity associated with them decreases rapidly with downstream distance. In fact, no significant levels of vorticity exist in the central region of the channel downstream of the valve beyond approximately $8 \mathrm{~mm}$.

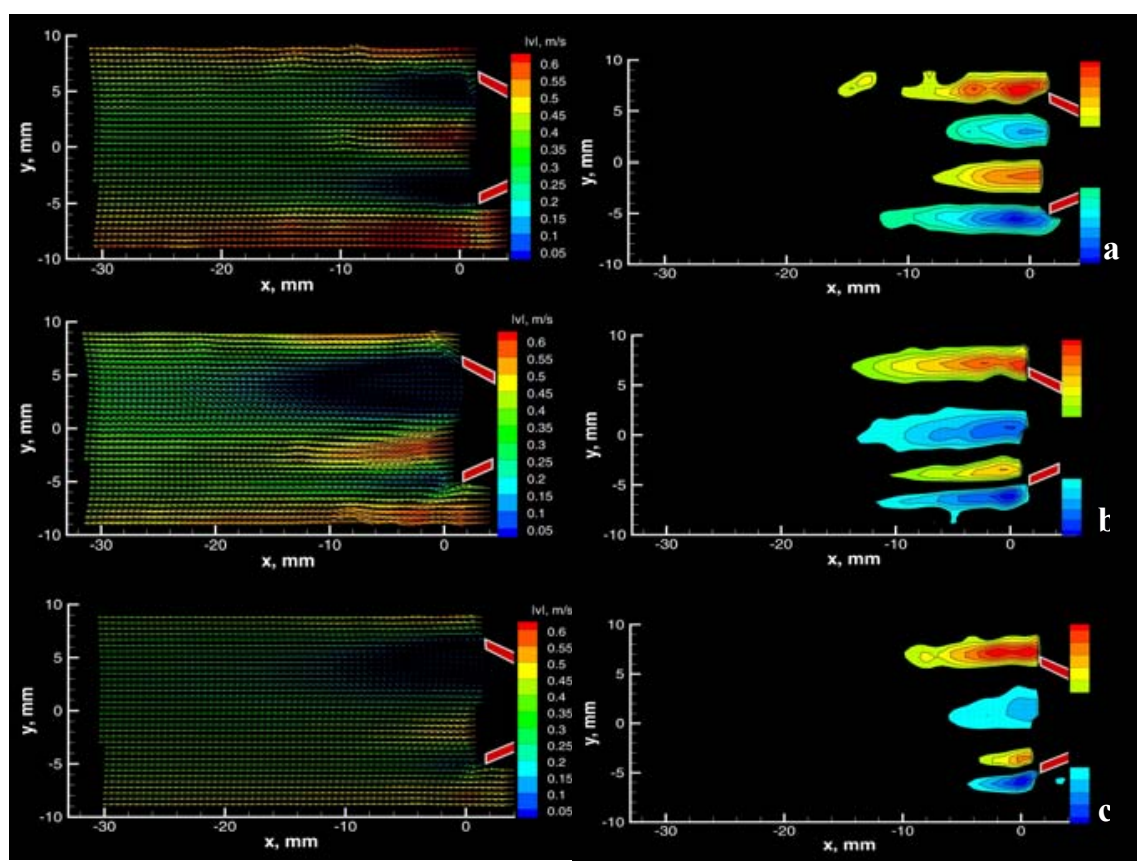

Figure 5: Time-average velocity field (left) and corresponding out-of-plane vorticity (right) for: (a) DAP C (b) DAP B (c) Dap C.

The time-averaged velocity field and the corresponding out-of-plane vorticity plot for DAP B are shown in Figure 5(b). Once again, the dominant feature of the flow field downstream of the valve is the presence of four separated shear layers that originate at the tips of the leaflets. However, the central jet has shifted toward the lower leaflet causing an asymmetry in the flow structure. The inner and outer shear layers that define the relatively large upper wake are formed at the leading and trailing edges of the top leaflet. They are represented, in Figure 5(b), by high levels of out-of-plane vorticity that extend $14 \mathrm{~mm}$ downstream of the valve. In contrast, the relatively small lower wake, which is defined by the inner and outer shear layers formed at the leading and 
trailing edges of the lower leaflet, contains lower circulation levels that only extend $10 \mathrm{~mm}$ downstream of the valve. The shift in the central jet's position reduces the distance between the shear layers that define the lower wake, causing them to interact. This interaction results in reduced levels of circulation in the lower wake as well as higher Reynolds stresses.

DAP C, which corresponds to a further increase in the distance from the centerline of the channel, shows a very similar flow structure as that of DAP B. As can be seen in Figure 5(c), the upper wake dominates the lower wake and the central jet has shifted toward the lower leaflet. In comparison to the results of DAP B, the overall velocity magnitude is lower for DAP C due to the proximity to the channel walls. Consequently, the size of both the upper wake and the lower wake decreases by the same amount.

\section{Conclusions}

Particle image velocimetry was used to investigate the flow structure downstream of a bileaflet mechanical heart valve (MHV) during peak systole. The data was obtained for three longitudinal cross-sections of the flow field, referred to as data acquisition planes (DAPs) A through C. In all three DAPs, the overall flow structure consisted of four separated shear layers containing vortices shed from the leading and trailing edge of each leaflet. These shear layers define two wakes and a high-velocity jet-like region located in the centre of the channel. Contrary to the traditional assumption of the dominant role of the outer shear layers, it was shown that the large-scale transverse oscillations of the inner shear layers dominate the near-wake of the valve. The large-scale flow oscillation corresponds to transverse undulations of the jet-like flow through the central opening of the valve and to flapping of the associated inner shear layers. These oscillations were characterized in terms of three representative flow regimes. Limited temporal resolution of the DPIV imaging sequence did not allow for accurate estimation of the large-scale oscillation frequency.

The flow directly downstream of the bileaflet MHV was found to be highly three-dimensional. Marked differences were found in the flow structure downstream of the MHV at each DAP. Predominantly, the central jet shifts position along the vertical axis, causing an increase in the size of one wake and a reduction in size of the other. In general, the shift of the central jet causes a significant change in the unsteady flow structures present downstream of the valve. The unsteady flow characteristics, in particular the Reynolds stresses, are of special importance due to their close link to platelet activation. Future investigations will focus on a quantitative characterization of the unsteady flow structures and the mechanisms of their interaction.

\section{References}

[1] A .P. Yoganathan, He, Z. and Jones, "Fluid Mechanics of Heart Valves", Annual Review of Biomedical Engineering, Vol. 6, 331-362 2004. 
[2] W. Yin, Alemu, Y., Affeld, K., Jesty, J. and Bluestein, D., "Flow-induced platelet activation in bileaflet and monoleaflet mechanical heart valves." Annals of Biomedical Engineering, Vol. 32, 8, 1058-1066, 2004.

[3] D. Bluestien, Yin, W., Affeld, K. and Jesty, J., "Flow- induced Platelet Activation in Mechanical Heart Valves" Journal of Heart Valve Disease, Vol. 13, 501-508, 2004.

[4] L.H. Edmunds, Mckinlay, S., Anderson, J. M., Callahan, T. H., Chesebro, J. H., Geiser, E. A., Makanani, D. M., McIntire, L. V., Meeker, W. Q., Naughton, G. K., Panza, J. A., Schoen, F. J. and Didisheim, P., "Directions for improvement of substitute heart valves: National Heart, Lung, and Blood Institute's Working Group report on heart valves." Journal of Biomedical Material Research, Vol. 38, 3, 263-266, 1997.

[5] Y.-R. Woo, and A. P. Yoganathan, "In-Vitro Fluid Dynamic Characteristics of the Abiomed Trileaflet Heart Valve Prosthesis" ASME, Vol. 105, 338-345, 1983.

[6] L.N. Scotten and Walker, D. K., "New Laboratory Technique Measures Projected Dynamics Area of Prosthetic Heart Valve" Journal of Heart Valve Disease, Vol. 13, 1, 120-133, 2004.

[7] M. Marassi, Castellini P., Pinotti, M., and Scalise, L. "Cardiac valve prosthesis flow performances measured by a 2-D and 3-D-stereo particle image velocimetry" Experiments in Fluids, Vol. 36, 1, 176-186, 2004.

[8] P. Castellini, Pinotti, P., and Scalise, L. "Particle Image Velocimetry for Flow Analysis in Longitudinal Planes across a Mechanical Artificial Heart Valve" Artificial Organs, Vol. 28, 5, 507-513, 2004.

[9] D. Bluestein, Rambod, E. and Gharib, M., "Vortex Shedding as a Mechanism for Free Emboli Formation in Mechanical Heart Valves" Journal of Biomedical Engineering, Vol. 122, 125-134, 2000.

[10] T.C. Lamson, Rosenberg, G., Geselowitz, D. B., Deutsch, S., Stinebring, D. R., Frangos, J. A. and Tarbell, J. M., "Relative Blood Damage in the Three Phases of a Prosthetic Heart Valve Flow Cycle" ASAIO Journal, Vol. 39, 3, M626-M633, 1993.

[11] K.B. Manning, Kini, V., Fontaine, A. A., Deutsch, S. and Tarbell, J., "Regurgitant Flow Field Characteristics of the St. Jude Bileaflet Mechanical Heart Valve under Physiological Pulsatile Flow Using Particle Image Velocimetry" Artificial Organs, Vol. 27, 9, 840-846, 2003.

[12] R.S. Meyer, Deutsch, S., Bachmann, C. B. and Tarbell, J. M., "Laser Doppler Velocimetry and Flow Visualization Studies in the Regurgitant Leakage Flow Region of Three Mechanical Mitral Valves.” Artificial Organs, Vol. 25, 4, 292-299, 2001. 